\title{
RESULTS OF WORK OF NEW SPECTROPOLARIMETER FOR SOLAR RADIO EMISSION OBSERVATIONS IN THE RANGE 50-500 MHz
}

\author{
N.O. Muratova \\ Institute of Solar-Terrestrial Physics SB RAS, \\ Irkutsk, Russia,muratova@iszf.irk.ru
}

\section{A.A. Muratov}

Institute of Solar-Terrestrial Physics SB RAS, Irkutsk, Russia, mutolya@mail.ru

\author{
L.K. Kashapova \\ Institute of Solar-Terrestrial Physics SB RAS, \\ Irkutsk,Russia,lkk@iszf.irk.ru
}

\begin{abstract}
Ground-based observations within meter radio range are of importance for understanding processes occurring in the solar corona.

We present concepts, block diagram, and results of first observations of the Solar Spectropolarimeter of Meter Range (SSMD), launched for solar observations in the range 50-500 MHz in April 2016. The main purpose of this work was to develop an up-to-date digital radio spectropolarimeter able to record the full Stokes vector for sporadic solar phenomena taking place in the 50-500 MHz range.

We use a crossed log periodic antenna to detect solar radio emission. This antenna can simultaneously obtain horizontal and vertical polarization components. The main part of SSMD is a digital receiver based on an FX correlator architecture. We use the Fast Fourier Transform (FFT) algorithm based on the real-time pipeline
\end{abstract}

\section{INTRODUCTION}

Accelerated particles in the solar corona are a source of a large number of phenomena occurring due to the interaction between magnetic fields and plasma. Nonthermal electrons accelerated in the solar atmosphere emit radio waves in a wide range and give rise to radio bursts of various types [Wild et al., 1963; Zheleznyakov, 1964; Kundu, 1965]. The study of events of this type has been of interest for decades. On the one hand, problems important for physics of the solar corona, which are associated with understanding the nature of radio bursts, still remain unsolved. On the other hand, current methods enable us to estimate plasma parameters during events associated with such geoeffective phenomena as coronal mass ejections or electron flux enhancement in solar cosmic rays [Tsvetkov et al., 2018]. The phenomena in this range are examined using the so-called dynamic spectra representing the distribution of values of a parameter as a function of time and frequency. The meter-wave emission of most known radio bursts is polarized [Zheleznyakov, 1964; McLean et al., 1985], and full information on the emission polarization is provided by the full Stokes vector [Born, Wolf, 1965]. Currently, spectroscopic observations at a meter range are made using such instruments as Culgoora (18-1800 $\mathrm{MHz}$, Australia) [Prestage et al., 1994]; Learmonth (25$180 \mathrm{MHz}$, Australia) [https://www.sws.bom.gov.au/ Solar/3/1]; Hiraiso (25-2500 MHz, Japan) [Kondo et al., 1995]; AMATERAS (150-500 MHz, Japan) [Iwai et al., 2012]; IZMIRAN spectrograph (25-270 MHz, Russia) circuit to construct amplitude dynamic spectra (intensity vs frequency and time).

SSMD has 4608 frequency channels with $97.66 \mathrm{kHz}$ channel bandwidth and $97.66 \mathrm{kHz}$ spacing. Time resolution is $1 \mathrm{~s}$. The spectropolarimeter has a $50-500 \mathrm{MHz}$ range. It can record the full Stokes vector. At present, SSMD observes two of four parameters in regular mode ( $I$ and $V$ ). We have observational data since 2016. The cata$\log$ development is in progress.

We plan to improve time and frequency characteristics, record all Stokes parameters, and conduct a calibration. We are working on providing access to the data archive via the Internet.

Keywords: radio spectrometer, Fast Fourier Transform spectrometer, FX correlator, instruments, Sun, polarization, Stokes parameters, meter wavelengths.

[http://www.izmiran.ru/stp/lars], etc. In addition to individual instruments there is the International Network of Solar Radio Spectrometers e-CALLISTO making observations in VHF and UHF bands. This network includes radio spectrographs with a frequency range 45$870 \mathrm{MHz}$, located in different parts of the globe. One of the e-CALLISTO spectrographs [Benz et al., 2009] performs observations at the Radio-astrophysical Observatory (Badary). This analog spectrograph measures, however, only the intensity of the horizontal polarization component. Most radio spectrographs observe either one polarization component (linear or circular) of the electric field vector or one Stokes parameter; even more rarely they observe two parameters. This is due to the fact that observations of the full Stokes vector impose special conditions on characteristics of the receiver, particularly on the ratio of channel width to observed frequency, requiring high sensitivity. The VHF range is also sensitive to ionospheric effects, and, moreover, is contaminated by signals from other numerous radio devices. The rapid development of electronics and the appearance of new affordable mass-produced microcircuits and processes enable progress in solving the problem. We report the results of the first stage of development and beginning of regular observations of the Solar Spectropolarimeter of Meter Range (50-500 MHz) (SSMD). This paper presents concepts of constructing the spectropolarimeter, discusses its digital and analog parts, the instrument's characteristics and observations obtained at the first stage. In conclusion, we present plans and objectives of improving the new instrument. 


\section{BASIC CONCEPT OF SSMD}

We aimed at designing a radio spectropolarimeter able to measure all Stokes parameters $(I, Q, U, V)$ of the solar radio emission observed in the range 50-500 $\mathrm{MHz}$. These measurements provide a full description of the state of radio emission polarization. Currently there are no similar radio spectrographs with such characteristics.

As a receiving antenna of SSMD we utilize a crossed 2x16-element log periodic antenna [http://www.cd-corp.com/eng/cma/clp5130.pdf] (Figure 1, $a, b)$. It features high strength, low cost and can cover a wide frequency range. Another advantage of this antenna is that it can simultaneously receive horizontal and vertical polarization components. Represent the plane electromagnetic wave incident on the antenna as two orthogonal components of the electric field vector:

$$
\begin{aligned}
& E_{x}=a_{1} \cos \left(\tau+\delta_{1}\right), \\
& E_{y}=a_{2} \cos \left(\tau+\delta_{2}\right) .
\end{aligned}
$$

From amplitudes $a_{1}, a_{2}$ and phase difference $\delta=\delta_{1}-\delta_{2}$ of Expression (1) we can determine Stokes vector parameters [Born, Wolf, 1965]. For quasi-monochromatic waves, these parameters take the following form:

$$
\begin{aligned}
& I=\left\langle a_{1}^{2}\right\rangle+\left\langle a_{2}^{2}\right\rangle, Q=\left\langle a_{1}^{2}\right\rangle-\left\langle a_{2}^{2}\right\rangle, U=2\left\langle a_{1} a_{2} \cos \delta\right\rangle, \\
& V=2\left\langle a_{1} a_{2} \sin \delta\right\rangle .
\end{aligned}
$$

For further calculations it is more convenient to use an equivalent notation, so we express these parameters through coherency matrix elements

$$
\begin{aligned}
& I=\left\langle E_{x} E_{x}^{*}\right\rangle+\left\langle E_{y} E_{y}^{*}\right\rangle, Q=\left\langle E_{x} E_{x}^{*}\right\rangle-\left\langle E_{y} E_{y}^{*}\right\rangle, \\
& U=\left\langle E_{x} E_{y}^{*}\right\rangle+\left\langle E_{y} E_{x}^{*}\right\rangle, V=i\left(\left\langle E_{x} E_{y}^{*}\right\rangle-\left\langle E_{y} E_{x}^{*}\right\rangle\right) .
\end{aligned}
$$

Here, $E_{x}$ and $E_{y}$ are analytical signals, $*$ is the complex conjugate, \langle\rangle is the time averaging, $I$ is the total intensity, $Q$ is the difference between intensities of $0^{\circ}$ and $90^{\circ}$ linear components, $U$ is the difference between intensities of $45^{\circ}$ and $135^{\circ}$ linear components, $V$ is the difference between intensities of left and right circular polarizations. SSMD

Next, we dwell on the analog and digital parts of

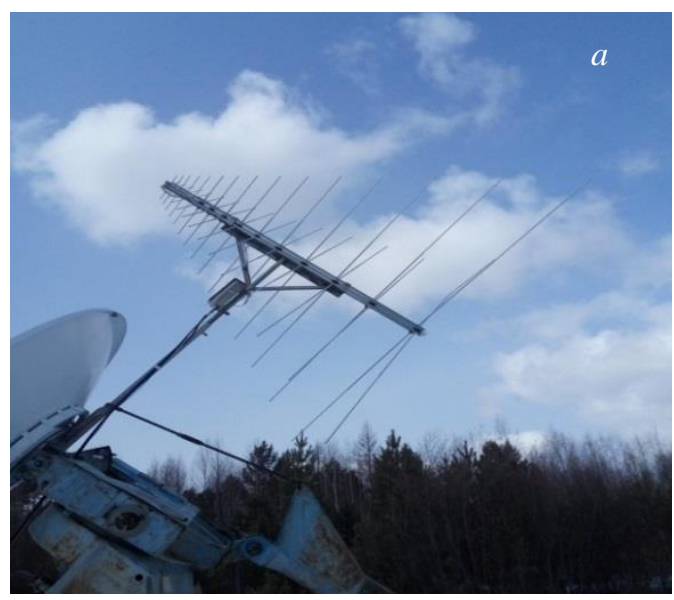

\section{SSMD ANALOG PART}

The SSMD electronics can be conveniently divided into two blocks. The first block is installed on the antenna mast in the hermetically closed box (see Figure 1, a) and contains analog electronics. The antenna (ANT) receives simultaneously the horizontal and vertical polarization components in the $50-500 \mathrm{MHz}$ range with the antenna gain 10-12 dB. Then (block diagram in Figure 2), the amplifier cascade amplifies these signals by $65 \mathrm{~dB}$ (AMPs). Hereinafter a calibration circuit is meant to be used which comprises two RF switches (SW), a noise generator (NG), and RF splitter (Split) and is located in the same box.

The amplified signals through coaxial 20-m cables arrive at the second block of SSMD comprising the second part of the analog circuit and digital electronics. The second block is located in the tunnel under the SSMD antenna. For further processing (Figure 2, BLOCK 2 (analog part)) we down-convert the $E_{x}$ and $E_{y}$ signals, using a superheterodyne circuit; the $50-500 \mathrm{MHz}$ range split into 10 subbands processed sequentially by the digital electronics. We down-convert our range because the sample rate of signals in the digital electronics is $100 \mathrm{MHz}$ (the maximum sample rate of the Analog-to-Digital Converter (ADC) is $125 \mathrm{MHz}$ ). The down conversion circuit includes one local oscillator (LO, GEN block) represented by an evaluation board with the frequency synthesizer EVALADF4350EB1Z [https://www.analog.com/en/index.html], controlled and tuned by the Field-Programmable Gate Array (FPGA), using a three-wire Serial Peripheral Interface (SPI). Then, the signal generated by the frequency synthesizer split for the $E_{x}$ and $E_{y}$ channels. In addition to the desired signal, the synthesizer generates the second, third, or other high-order harmonics. To avoid the effect of these harmonics, we add a board with the low-pass filter matrix (LPFM) to the output of the frequency synthesizer board; LPFM is assembled by surface mount components - filters and two RF switches responsible for the selection of the filter required for a given frequency band.

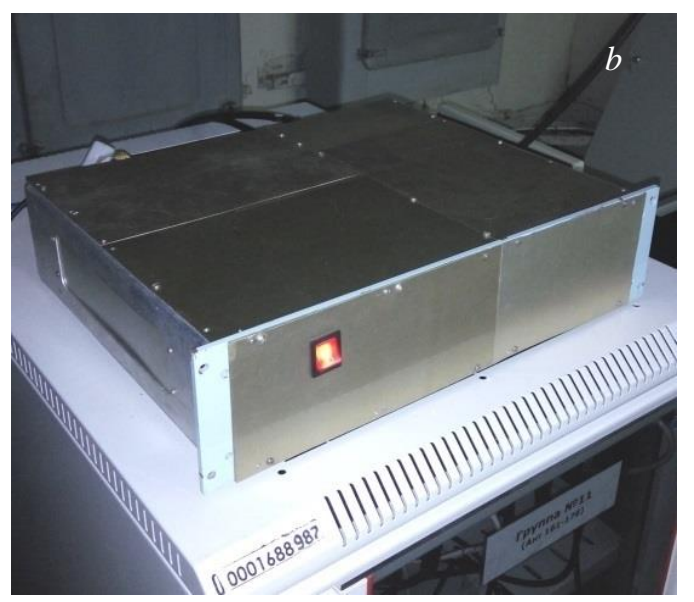

Figure 1. Crossed 2x16-element log periodic antenna CLP5130-1X, on the mast of which a hermetically closed box is fixed which contains a block of SSMD analog electronics (a); SSMD block (exterior) having an analog and digital electronics and located in the tunnel under the SSMD antenna $(b)$ 


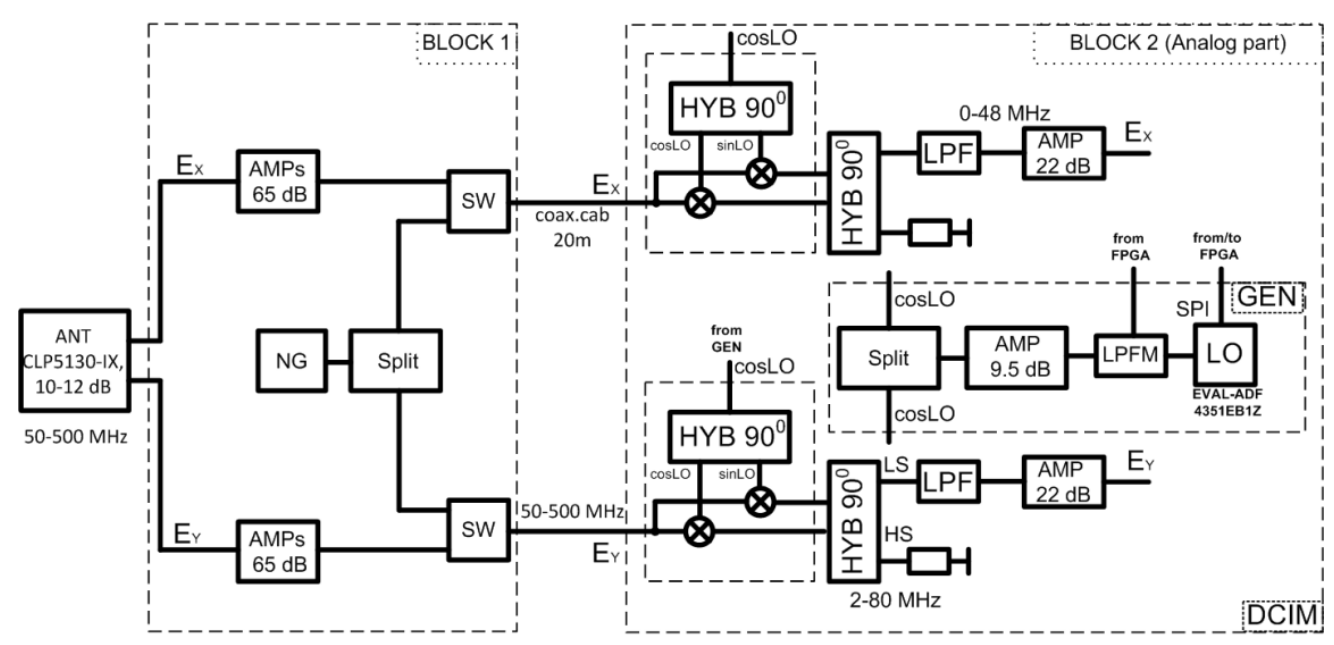

Figure 2. Block diagram of the SSMD analog part

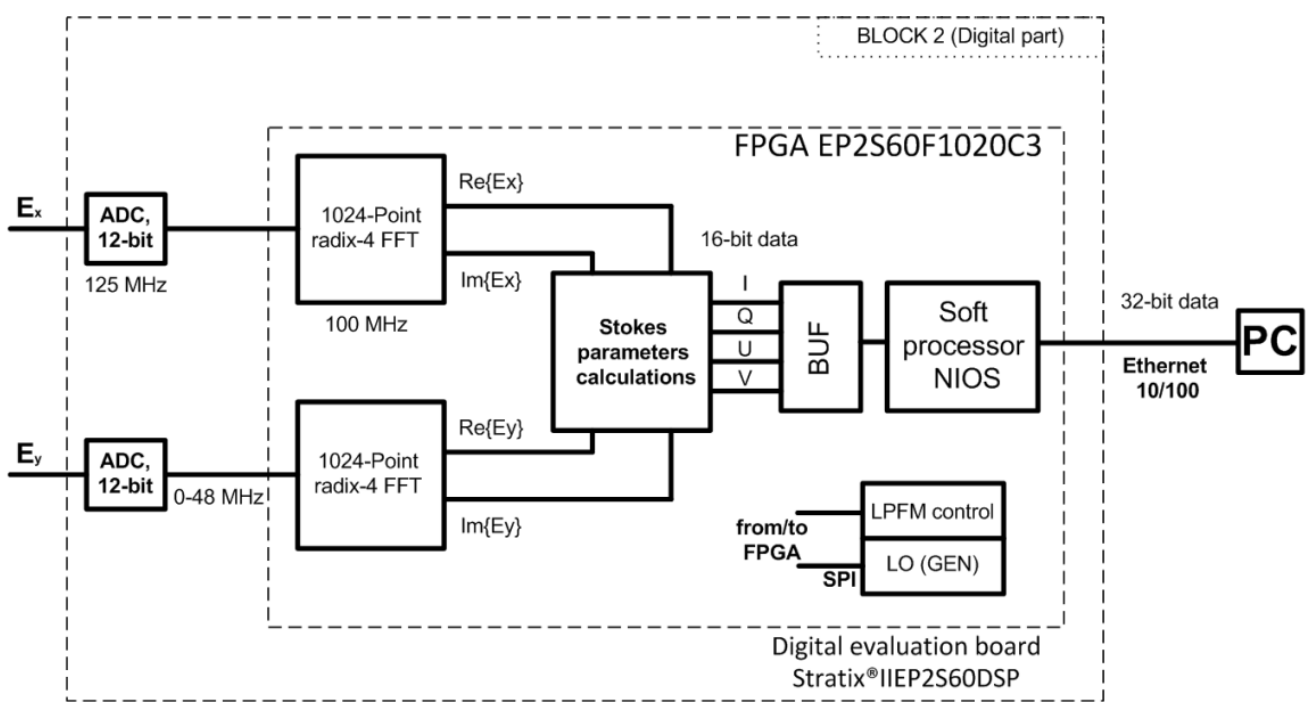

Figure 3. Block diagram of the SSMD digital part

The LPFM block is controlled using FPGA. When mixing signals at the mixer output, along with the desired lower-frequency signal we have an image channel. To reject it, in the down conversion circuit (DCIM block) we apply the Hartley architecture [Hartley, 1928], which involves using two $90^{\circ}$ hybrids (HYB) and two mixers. The input quadrature hybrid and two mixers are installed on one board - this prevents additional signal distortions. After the output quadrature hybrid, the frequency range of $E_{x}$ and $E_{y}$ is $2-80 \mathrm{MHz}$. Then these signals pass through a low-pass filter (LPF), after which their range is $2-48 \mathrm{MHz}$. Now the signals are ready for processing by the SSMD digital part, which is in one box with the above devices of the analog block of the circuit.

\section{SSMD DIGITAL PART}

The SSMD digital part is implemented on the digital evaluation board Stratix ${ }^{\circledR}$ IIEP2S60DSP produced by Altera (since June 2015, Intel Corporation [https://www. intel.com]). The $E_{x}$ and $E_{y}$ signals processed by the analog part are pre-amplified by $22 \mathrm{~dB}$ and then digitized by 12 bit ADC installed on the evaluation board (Figure 3). As mentioned above, the sample rate is $100 \mathrm{MHz}$ and the ob- served frequency range of SSMD is $50-500 \mathrm{MHz}$, therefore we have used the down conversion circuit. Bands of the digitized $E_{x}$ and $E_{y}$ signals of the previously split 50$500 \mathrm{MHz}$ range are $46 \mathrm{MHz}$, each such subband sequentially arrives at EP2S60F1020C3 FPGA, where it is processed by the Fast Fourier Transform (FFT) block operating at a clock frequency of $100 \mathrm{MHz}$. In addition to the digital signal processing, which is the main FPGA function, it controls and tunes the frequency synthesizer board through 3-wire SPI, controls the input ADC and LPFM, as well as data transmission via the Ethernet from the digital board to a personal computer (PC). Let us focus on the digital processing block.

Consider Expression (3). In nature, signals are real. We need to obtain analytic and complex conjugate components from the real signals. Further, according to (3), we need to find two autocorrelation functions (ACF) and two cross-correlation functions (CCF). The Wiener-Khinchin theorem states that the signal power spectrum $W(\omega)$ is related to its $\mathrm{ACF} B_{S}(\tau)$ by the Fourier transform [Baskakov, 2003; Wilson et. al., 2009]:

$$
W(\omega)=\int_{-\infty}^{\infty} B_{S}(\tau) e^{-j \omega \tau} d \tau,
$$


where

$$
\begin{aligned}
& W(\omega)=S(\omega) S^{*}(\omega), \\
& B_{S}(\tau)=\int_{-\infty}^{\infty} S(t) S^{*}(t-\tau) d t .
\end{aligned}
$$

The cross-power spectrum $W_{U V}(\omega)$ and CCF $B_{U V}(\tau)$ of two signals are similarly related by the Fourier transform:

$$
W_{U V}(\omega)=\int_{-\infty}^{\infty} B_{U V}(\tau) e^{-j \omega \tau} d \tau,
$$

where

$$
\begin{aligned}
& W_{U V}(\omega)=U(\omega) V^{*}(\omega), \\
& B_{U V}(\tau)=\int_{-\infty}^{\infty} U(\tau) V^{*}(t-\tau) d \tau .
\end{aligned}
$$

Using statements (4) and (5) when creating the SSMD digital processing block, we applied the FX-correlator architecture [Romney, 1999].

First we obtain signal spectra and then calculate Stokes components. To calculate the $E_{x}$ and $E_{y}$ spectra, we implement the 1024-point radix-4 FFT algorithm in FPGA. Taking into account characteristics of devices of the analog circuit, we use 471 (and 369 for the last subband) frequency channels of 512 FFT calculated for each subband. Accordingly, for the $50-500 \mathrm{MHz}$ range we obtain 4608 channels. This algorithm is realized using a pipeline architecture [Rabiner et al., 1975] and operates in real time, thus allowing us to process input signals without data loss. Given (3)-(5), we obtain Stokes parameters and accumulate them (BUF). The accumulated 32-bit data is transferred from the evaluation board to PC via LAN Ethernet 10/100. For the data transfer we use NIOS microprocessor [https://www.intel.com], which is also part of the FPGA firmware. The microprocessor is controlled by the software package created in Eclipse IDE using the $\mathrm{C}$ programming language. The firmware for FPGA is implemented using VerilogHDL with the software (SW) QuartusII [https://www.intel.com] and occupies $20 \%$ of logical resources of the EP2S60F1020C3 FPGA and $30 \%$ of memory resources. SW for displaying data on PC and their record is made in Qt SDK (Software Development Kit [https://www.qt.io]) using C.

\section{MAIN PARAMETERS OF SSMD}

Table lists the main parameters of SSMD. As mentioned in previous sections, to process the $50-500 \mathrm{MHz}$ range on the basis of $100 \mathrm{MHz}$ FPGA sample rate, we split it into $46 \mathrm{MHz}$ subbands. Note that initially the width of each subband to be processed by FFT is 50 $\mathrm{MHz}$, but due to characteristics of the analog circuit we overlap the subbands and reduce the width to $46 \mathrm{MHz}$ (36 MHz for the last one). Thus, of the 512 channels calculated using FFT, we use 471 (and 369 for the last one) channels for each subband, the full spectrum comprises 4608 channels. The channel width is $97.66 \mathrm{kHz}$ with $97.66 \mathrm{kHz}$ spacing. At the input of the digital evaluation board after ADC, data has 12-bit resolution, thus the dynamic range of the digital part is $72 \mathrm{~dB}$. At the output of the digital part, the bit resolution of data is 32 bits. The time resolution of SSMD is $1 \mathrm{~s}$. It is reduced by such factors as the speed of devices on the digital board and Ethernet connection, the use of the sequential processing of the 50-500 $\mathrm{MHz}$ range instead of the direct one. SSMD calculates the full Stokes vector, but at present $I$ and $V$ (intensity and circular polarization) are recorded on PC. In the future, we plan to record all the four parameters. As previously noted [Zheleznyakov, 1964; Akabane et al., 1961; Chin et al., 1971], when observing the linear polarization $(Q$ and $U$ Stokes parameters) we should take into account the Faraday effect and compute the channel width so as to reduce the blurring effect of polarization in the channel, which is associated with the finite receiver band. Basing on the preliminary calculations and practical measurements carried out by other researchers, to increase the probability of detection of the linear polarization, we took the channel width to be $10 \mathrm{kHz}$. At the first stage of the development of the instrument, in order to increase the sensitivity we decided to take the channel width to be 97.66 $\mathrm{kHz}$, which allowed us to test and tune the instrument for such important parameters as total intensity and circular polarization. The size of recorded data is $1.2 \mathrm{~GB}$ per day; the data is available in binary format. At present, the work on their hosting on the Institute server is in progress.

Main parameters of SSMD

\begin{tabular}{|l|c|}
\hline \multicolumn{1}{|c|}{ Parameter } & Value \\
\hline frequency range & $50-500 \mathrm{MHz}$ \\
\hline number of channels & 4608 \\
\hline subband width & $46 \mathrm{MHz}$ \\
\hline number of channels in the subband & $471(369 \mathrm{for}$ the last one $)$ \\
\hline channel width (spacing) & $97.66 \mathrm{kHz}(97.66 \mathrm{kHz})$ \\
\hline Sample rate & 12 bit $(32 \mathrm{bit})$ \\
\hline bit resolution of input (output) data & full Stokes vector $(I, Q, U, V)$ \\
\hline type of measured polarization & 1 and $V$ components are recorded $)$ \\
\hline time resolution & $00: 00-10: 00 \mathrm{UT}$ \\
\hline time span of observations & $1.2 \mathrm{~GB}$ \\
\hline data recorded per day &
\end{tabular}




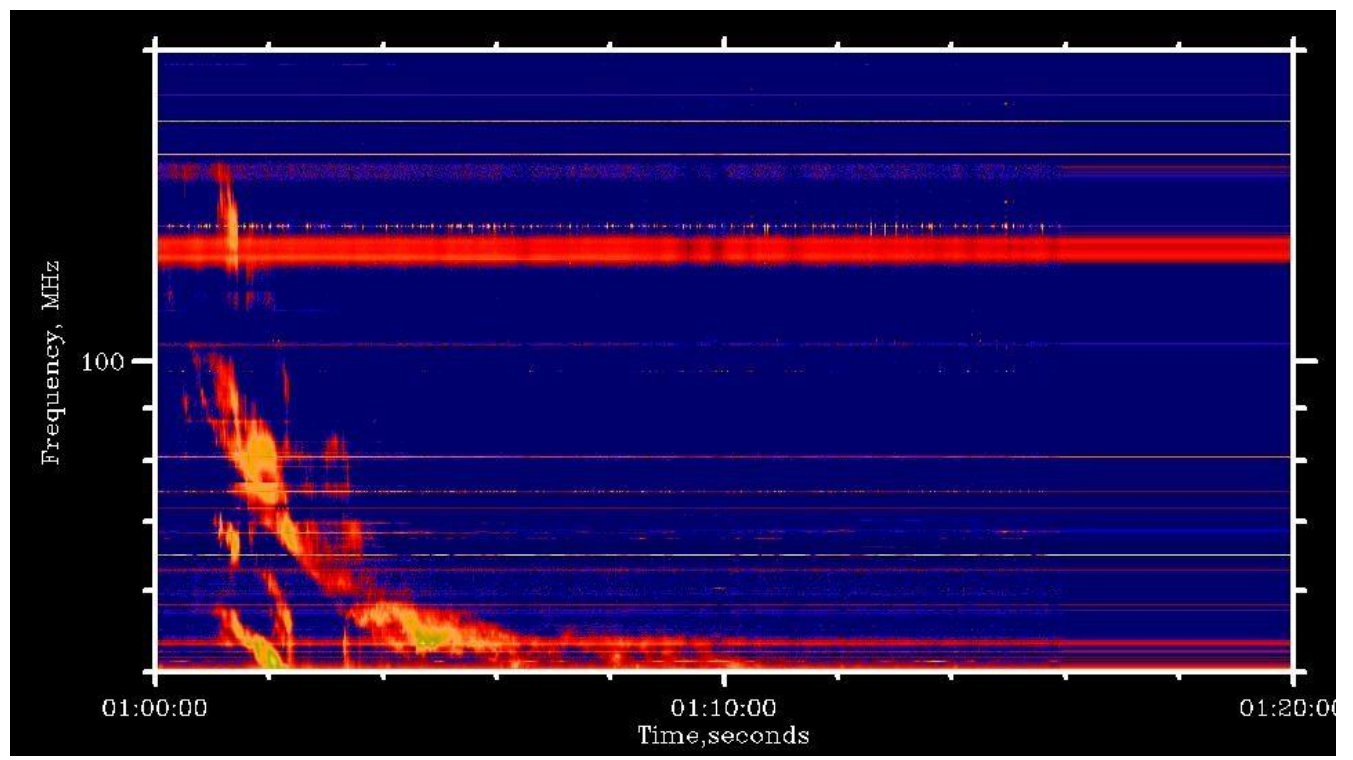

Figure 4. Type II radio burst (intensity I) recorded by SSMD on July 10, 2016
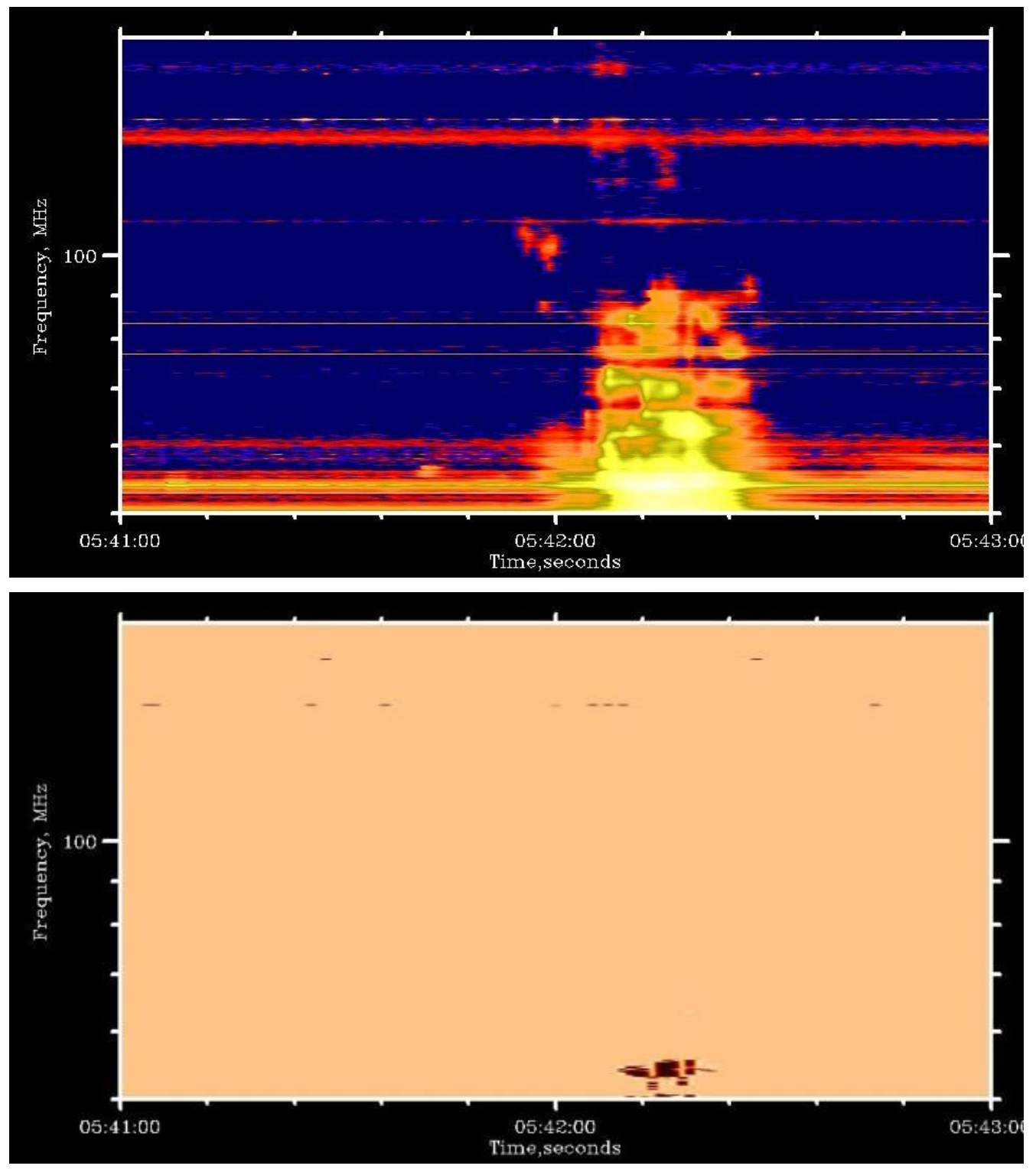

Figure 5. Type III radio burst recorded by SSMD on July 23, 2016: intensity $I(a)$; circular polarization $V(b)$ 


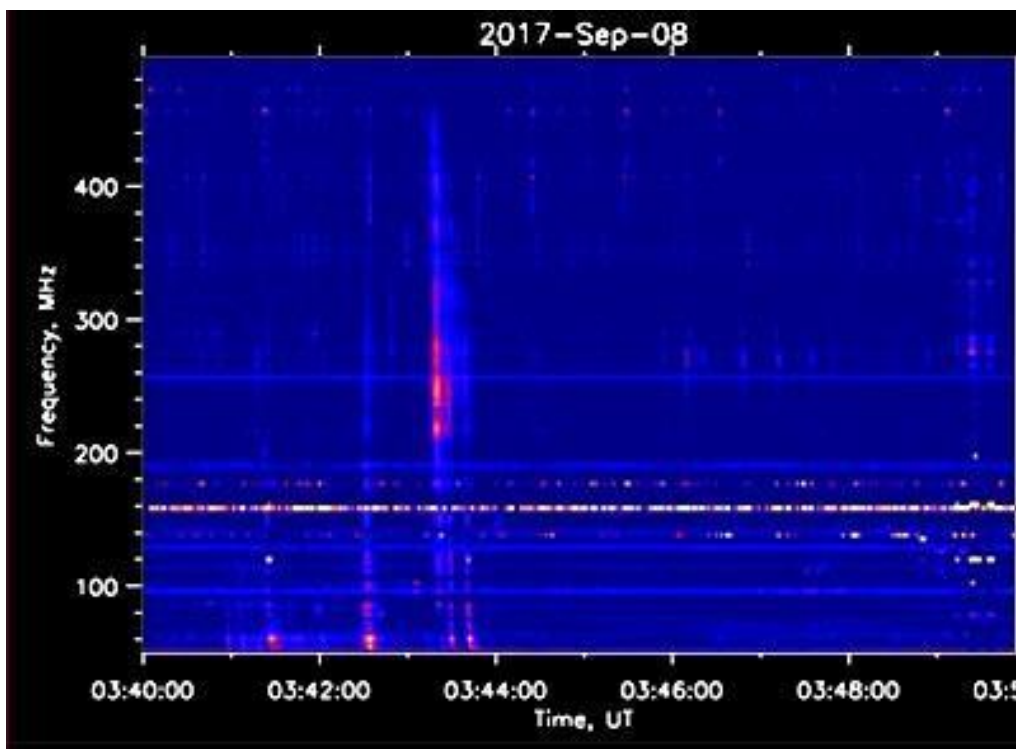

Figure 6. Type III radio burst ( $I$ intensity spectrum ) recorded by SSMD on September 08, 2017

\section{FIRST OBSERVATIONAL RESULTS}

The instrument has successfully started regular observations at the ISTP SB RAS Radioastrophysical Observatory ( Badary) in April 2016. One of the first events recorded with the instrument was a radio burst with slow frequency drift, or type II radio burst, observed during a solar flare on July 10 , 2016 (see Figure 4). During another solar flare on July 23, 2016, we detected radio bursts with fast frequency drift, or type III bursts. SSMD also detected the presence of circular polarization. Dynamic spectra of these two parameters are shown in Figure 5. The above events are not artifacts - this is confirmed by the fact that they were also recorded by eCALLISTO instruments and by the radio spectrograph of Learmonth Observatory [https://www.sws.bom.gov.au/ Solar/3/1] (Australia). Observations during higher solar activity in September 2017 have shown that the spectrograph has sufficient sensitivity throughout the given range (Figure 6).

Only the $I$ intensity spectra are currently available for analysis. Observations of circular polarization (Stokes parameter $V$ ) are still tested to specify the sign of polarization. Due to the regular observations, we have collected sufficient material to catalog radio bursts observed by SSMD in 2016-2018.

\section{CONCLUSION}

An original instrument has been developed, built, and has started regular observations. It provides dynamic spectra of solar radio emission in the range 50-500 $\mathrm{MHz}$ with a time resolution of $1 \mathrm{~s}$ and a channel bandwidth of $97.66 \mathrm{kHz}$. The first observations with this new instrument have shown that it can detect radio bursts having various dynamic and brightness characteristics. In the near future, we plan to make observations of circular polarization available for scientific analysis. We also plan to make regular observations of the full Stokes vector and to improve characteristics of the spectrograph, using experience gained from the first observations.

The work was performed with budgetary funding of Basic Research program II.16.

\section{REFERENCES}

Akabane K., Cohen M.H. Polarization measurements of type III bursts and Faraday rotation in the corona. Solar Phys. 1961, vol. 133, pp. 258-268

Baskakov S.I. Radiotekhnicheskie tsepi i signaly [Radio circuits and signals]. Moscow, Vysshaya Shkola Publ., 2003. 462 p. (In Russian).

Benz A.O., Monstein C., Meyer H., Manoharan P.K., Ramesh R., Altyntsev A., et al. A world-wide net of solar radio spectrometers: e-CALLISTO. Earth, Moon, and Planets. 2009 , vol. 104 , iss. $1-4$, pp. $277-285$. 10.1007/s11038-0089267-6.

Born M., Wolf E. Principles of Optics. London: Pergamon Press, 1965, 808 p.

Chin Y.C., Lusignan B.B., Fung P.C.W. Polarization measurements of solar type III radio bursts at $25.3 \mathrm{MHz}$. Solar Phys. 1971, vol. 16, iss. 1, pp. 135-151. 10.1007/BF00154509.

Hartley R. Modulation System. US patent, no. 1666206, 1928.

Iwai K., Tsuchiya F., Morioka A., Misawa H. IPRT/AMATERAS: A new metric spectrum observation system for solar radio bursts. Solar Phys. 2012, vol. 277, iss. 2, pp. 447-457. 10.1007/s11207-011-9919-y.

Kondo T., Isobe T., Igi S., Watari S., Tokimura M. The Hiraiso Radio Spectrograph (HiRAS) for monitoring solar radio bursts. J. Communications Research Laboratory. 1995, vol. 42, no. 1, pp. 111-119.

Kundu M. R. Solar Radio Astronomy. N.Y.: Interscience Publ., 1965. 660 p.

McLean D.J., Labrum N.R. Solar Radiophysics: Studies of Emission from the Sun at Metre Wavelengths. N.Y.: Cambridge University Press, 1985, 516 p.

Prestage N.P., Luckhurst R.G., Paterson B.R., Bevins, C.S., Yuile, C.G. A new radiospectrograph at Culgoora. Solar Phys. 1994, vol.150, pp. 393-396. DOI: 10.1007/BF00712901.

Rabiner L.R., Gold B. Theory and Application of Digital Signal Processing. New Jersey: Englewood Cliffs, PrenticeHall, Inc. Publ, 1975, 777 p.

Romney J.D. Cross-correlators. Synthesis imaging in radio astronomy II. A collection of lectures from the Sixth NRAO/NMIMT Synthesis Imaging Summer School. ASP Conf. Ser. Socorro, New Mexico, USA, 1999, vol. 180, pp. 57-78.

Tsvetkov Ts., Miteva R., Petrov N. On the relationship between filaments and solar energetic particles. J. Atmos. SolarTerr. Phys. 2018, vol. 179, pp. 1-10. DOI: 10.1016/j.jastp. 2018.06.005. 
Wild J.P., Smerd S.F., Weiss A.A. Solar Bursts. Annual Review of Astronomy and Astrophysics. 1963, vol. 1, no. 1, pp. 291-366. DOI: 10.1146/annurev.aa.01.090163.001451.

Wilson T.L., Rohlfs K., Hüttemeister S. Tools of Radio Astronomy. Berlin: Springer-Verlag Publ. 2009, 520 p.

Zheleznyakov V.V. Radio Emission of the Sun and Planets. Oxford, Pergamon Press, 1970, 712 p. (ed.: J.S. Hey).

URL: https://www.analog.com/en/index.html (accessed 30 July 2019).

URL: https://www.intel.com (accessed 30 July 2019).

URL: https://www.sws.bom.gov.au/Solar/3/1 (accessed 30 July 2019).
URL: http://www.cd-corp.com/eng/cma/clp5130.pdf (accessed 30 July 2019).

URL: http://www.izmiran.ru/stp/lars/?LANG=ru (accessed 30 July 2019).

URL: https://www.qt.io (accessed 30 July 2019).

How to cite this article

Muratova N.O., Muratov A.A., Kashapova L.K. Results of work of new spectropolarimeter for solar radio emission observations in the range 50-500 MHz. Solar-Terrestrial Physics. 2019. Vol. 5. Iss. 3. P. 3-9. DOI: $10.12737 /$ stp-53201901. 